\title{
Correlated barrier hopping in NiO films
}

\author{
P. Lunkenheimer and A. Loidl \\ Institut für Physik, Johannes Gutenberg-Universität, D-6500 Mainz, Federal Republic of Germany \\ C. R. Ottermann and K. Bange \\ Schott Glaswerke, Research and Development, P.O. Box 2480, D-6500 Mainz, Federal Republic of Germany
}

(Received 26 April 1991)

\begin{abstract}
The ac conduction in $\mathrm{NiO}$ films has been investigated in the frequency range $10 \mathrm{~Hz}<v<10^{9} \mathrm{~Hz}$ and at temperatures between 10 and $300 \mathrm{~K}$. The frequency and the temperature dependence of the electrical conductivity can be consistently explained within a model developed for the mechanism of charge transfer in amorphous semiconductors which proposes that charge carriers hop over potential barriers between defect sites, the height of the barriers being correlated with the intersite separation.
\end{abstract}

Nickel oxide is a Mott-Hubbard insulator ${ }^{1}$ which crystallizes in a high-temperature rocksalt structure and exhibits antiferromagnetism below $523 \mathrm{~K} .{ }^{2}$ Below the Néel temperature the lattice contracts along one of its body diagonals and undergoes a cubic to rhombohedral phase transformation. ${ }^{3} \mathrm{NiO}$ is a metal-deficient $p$-type semiconductor and the most thoroughly and most carefully studied narrow-band transition-metal oxide. However, despite a huge amount of conductivity, thermoelectric power and Hall mobility investigations it was impossible to arrive at definite conclusions concerning the electrical transport properties. The long-standing and persistent controversy, on whether the conduction in $\mathrm{NiO}$ is due to thermal excitation of the carrier concentration (band semiconductor) or due to thermal excitation of the mobility (small-polaron hopping) is documented in a number of review articles. ${ }^{4}$

Renewed interest arose due to the fact that hydrated $\mathrm{NiO}$ can be used as an active electrochromic material. There have been a number of proposals for the development of electrochromic devices such as displays, active optical filters, automotive rear-view mirrors with adjustable reflectance and smart windows with adjustable absorption and reflectance in the visible and near-infrared wavelength range. ${ }^{5}$

In this paper we report measurements of the frequency $v$ and temperature $T$ dependence of the ac conductivity $\sigma(v, T)$ in reactively evaporated $\mathrm{NiO}$ films. We will provide evidence that both the frequency and the temperature dependence of $\sigma$ can be well described in the correlatedbarrier hopping $(\mathrm{CBH})$ model. In this model it is assumed that the charge carriers hop between defect centers over the potential barrier $W$ separating them. The barrier height is correlated with the separation $R$ of the defect states. This model was introduced by Pike ${ }^{6}$ to account for the dielectric loss in scandium oxide films. With a similar model Pollak and Pike ${ }^{7}$ investigated the ac conductivity of glasses due to atomic and electronic hopping. Later on the $\mathrm{CBH}$ model was successfully applied by Elliott ${ }^{8}$ to explain the ac loss in chalcogenide glasses assuming that electron pairs hop between defect centers. In the singleelectron $\mathrm{CBH}$ model, the frequency dependence of the conductivity can be expressed by ${ }^{9,10} \sigma \sim \omega^{s}$, with $s=1-6 k_{B} T /\left[W_{m}-k_{B} T \ln \left(1 / \omega \tau_{0}\right)\right]$. The temperature dependence of $\sigma$ can, in the narrow-band limit, be given in power-law form as ${ }^{9}, 10 \sim T^{n}$, with $n=(1-s) \ln \left(1 / \omega t_{0}\right)$. $W_{m}$ is the barrier height for infinite intersite separation and thus corresponds to the energy to take the charge carrier from the defect state to the continuum (as we assume holes to be the charge carriers this is the upper edge of the valence band). $\tau_{0}$ is a characteristic relaxation time of the order of the inverse Debye frequency.

The nickel oxide films were deposited on glass substrates or on polished nickel sheets. The former samples were investigated in a coplanar geometry, the latter in a sandwich geometry. Due to the low conductivity of the samples investigated in these experiments reliable results could only be achieved in measurements using the sandwich geometry. Nickel oxide films were deposited by reactive electron-beam evaporation in a commercial deposition system (Balzers BAK 550) with a partial pressure of oxygen of $2 \times 10^{-2} \mathrm{~Pa}$. The substrate temperature was $300 \mathrm{~K}$ which increased during the evaporation process but was always below $400 \mathrm{~K}$. A deposition rate of $0.2 \mathrm{~nm} \mathrm{~s}^{-1}$ was used and the film thickness was $610 \mathrm{~nm}$. Due to the conditions of the evaporation process, spurious amounts of hydrogen cannot be excluded.

A broad spectrum of analytical methods was employed to characterize the as-deposited films. ${ }^{11}$ The chemical composition of the layers was $\mathrm{Ni}_{1}-\delta \mathrm{O}$ with $0<\delta<0.2$. These reactively evaporated $\mathrm{NiO}$ films can thus be characterized as metal deficient structures analogous to the findings in bulk materials. The density of the film was found to be $(65 \pm 5) \%$ of the bulk material. X-ray scattering investigations at room temperature revealed a polycrystalline structure of cubic (rocksalt-type) symmetry with $a=b=c=4.17 \AA$. A rhombohedral distortion of $\approx 0.1^{\circ}$, as found in bulk materials, ${ }^{3}$ is too small to be observed in these experiments.

Measurements of the dielectric permittivity were recorded using two different experimental set-ups: In the audio-frequency range automated low-frequency bridges (HP 4192A impedance analyzer and HP 4274A LCR meter) were used. These experiments were performed in home-manufactured variable-flow ${ }^{4} \mathrm{He}$ cryostats $(2 \mathrm{~K}$ $<T<300 \mathrm{~K})$. The high-frequency data were measured 
using a HP 4191A impedance analyzer connected to a refrigerator system (CTI Cryogenics) via an air line. This latter system is suitable for dielectric measurements in the frequency range from $1 \mathrm{MHz}$ to $1 \mathrm{GHz}$ and at temperatures between 10 and $450 \mathrm{~K} .{ }^{12}$

We recorded the complex capacitance $C^{*}$ which is related to the complex permittivity $\epsilon^{*}$ via $C^{*}(\omega, T)$ $=C_{0} \epsilon^{*}(\omega, T) . \quad C_{0}$ is the geometrical capacitance and $\omega=2 \pi \nu$ the angular frequency. In dielectric measurements of thin films in sandwich geometry it is difficult to adjust the two electrodes at a distance equal to the film thickness. That leads to an uncomplete filling of the capacitor and makes it impossible to determine the geometrical capacitance exactly. Thus we report our results in terms of the complex capacitance $C^{*}=C_{1}-i C_{2}$ or in terms of the real part of the conductance defined by $G=\omega C_{2}$. A rough estimate of the conductivity at room temperature, using the dielectric constant of the bulk material, yields $\sigma=6.5 \times 10^{-8} \Omega^{-1} \mathrm{~cm}^{-1}$ at $1 \mathrm{kHz}$. This value corresponds to the conductivity of pure single crystalline $\mathrm{NiO}$ samples. ${ }^{13}$

Figure 1 shows the temperature dependence of the conductivity in $\mathrm{NiO}$ as measured at various frequencies $v$ between $10 \mathrm{~Hz}$ and $1 \mathrm{GHz}$. In Fig. 1(a) the high-frequency data are plotted and (b) shows the audio-frequency re-

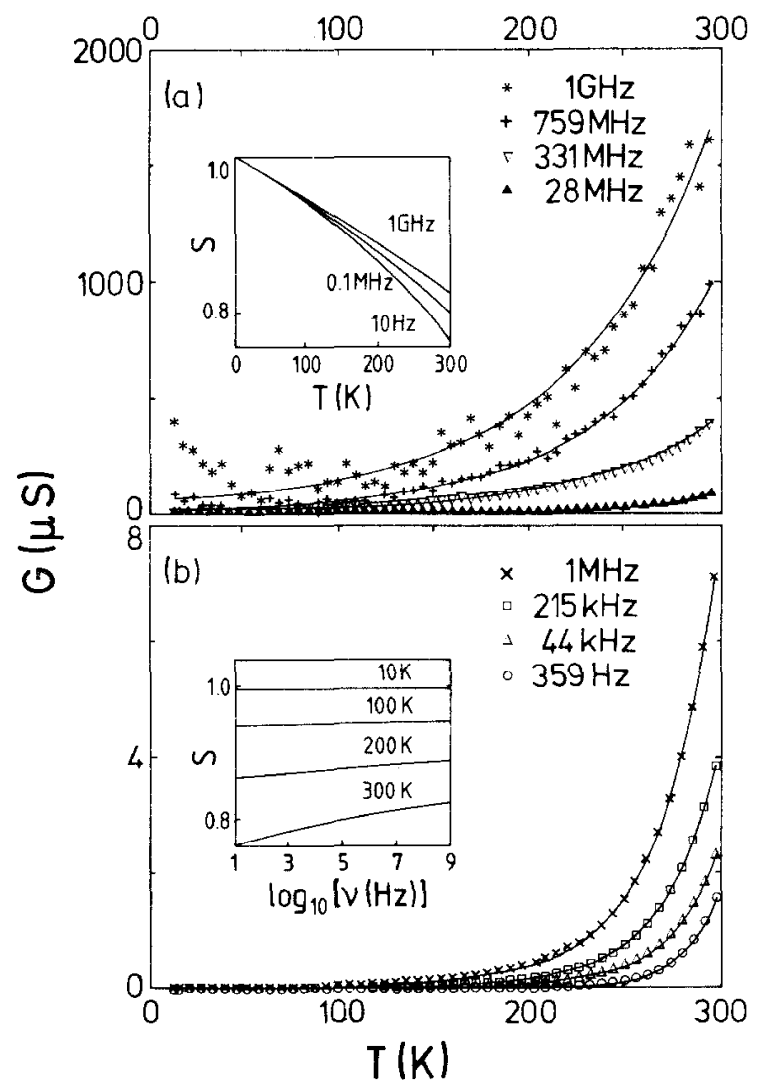

FIG. 1. Temperature dependence of the conductivity in $\mathrm{NiO}$ films at different measuring frequencies: (a): $1 \mathrm{GHz}(*) ; 759$ $\mathrm{MHz}(+) ; 331 \mathrm{MHz}(\nabla)$; and $28 \mathrm{MHz}(\underline{A}) ;(b)$ : $1 \mathrm{MHz}(x)$; $215 \mathrm{kHz}(\square) ; 44 \mathrm{kHz}(\triangle)$; and $359 \mathrm{~Hz}(\mathrm{O})$. The solid lines are calculated using the $\mathrm{CBH}$ model. The temperature dependence and the frequency dependence of the frequency exponent $s$ are shown as insets. sults. The experimental results were fitted assuming $G=A(\omega) T^{n}$ where $n=n(s, \omega, T)$ and $s=s(\omega, T)$ as determined from the CBH model indicated above. $W_{m}$ was the only fitting parameter. $\tau_{0}=10^{-12} \mathrm{~s}$ was fixed at a characteristic inverse phonon frequency. For all the measuring frequencies the fits to $G(T)$ yield an almost constant value of $W_{m} / k_{B}=22300 \pm 2000 \mathrm{~K}$ which corresponds to an energy difference between the ground state of the potential wells of the defect states and the band state of $1.9 \mathrm{eV}$. The results of the fits are shown as solid lines in Fig. 1. Using the average value of $W_{m}$ and the fixed $\tau_{0}$ we were able to calculate the frequency and temperature dependence of the frequency exponent $s$. Both dependencies, $s(T)$ at different measuring frequencies and $s(\omega)$ at different temperatures, are shown as insets in Figs. 1(a) and 1 (b), respectively.

Figure 2 shows the frequency dependence of the ac conductivity in $\mathrm{NiO}$ at various temperatures. Here we plotted $\log _{10} G$ vs $\log _{10} v$. At low frequencies the frequencyindependent conductivities signal the regime of pure dc conductivity $G_{\mathrm{dc}}$, while for higher frequencies the $\omega^{s}$ behavior of the ac conductance is nicely demonstrated. The experimental results were fitted using $G(\omega)=G_{\mathrm{dc}}+A \omega^{s}$ which is the usual approach to parametrize the experimentally observed conductance $G .{ }^{9,10}$ From the fits to the temperature dependence of $G$ we suggest that the dc conductance is simply the $\omega \rightarrow 0$ limit of the ac conductivity. In this case the validity of the separation used is dubious, but is nevertheless often used. ${ }^{9,10}$ At low temperatures $(T<200 \mathrm{~K})$ the resulting frequency exponents $s$ compare

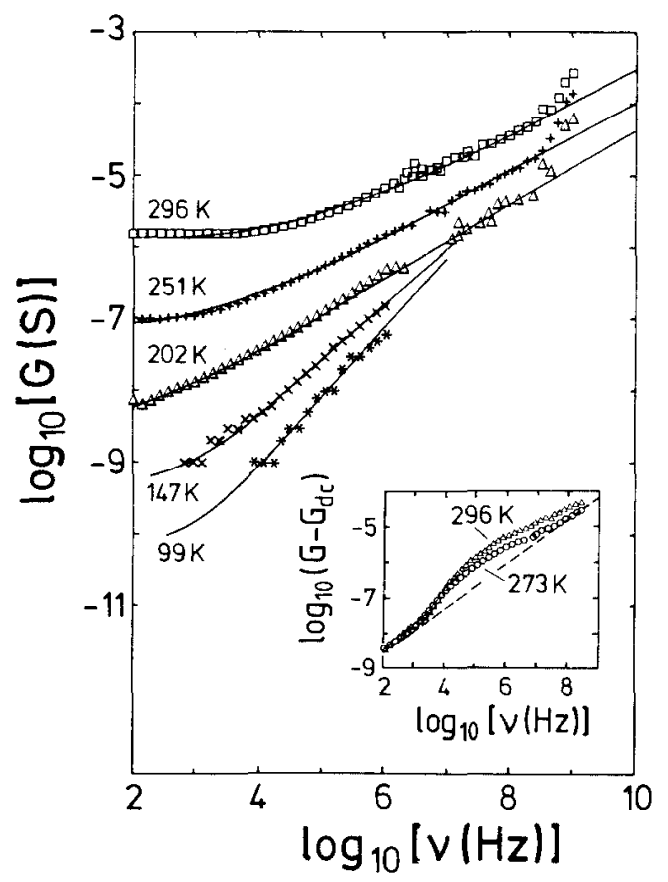

FIG. 2. Frequency dependence of the conductivity at different temperatures. $\log _{10} G$ is plotted vs $\log _{10} v: 296 \mathrm{~K}(\square) ; 251$ $\mathrm{K}(+) ; 202 \mathrm{~K}(\Delta) ; 147 \mathrm{~K}(x)$; and $99 \mathrm{~K}(*)$. The solid lines were calculated as described in the text. The inset shows the data at $296 \mathrm{~K}$ and at $273 \mathrm{~K}$ after subtraction of the dc conductivity. The dashed line is an estimate of the frequency dependence of the ac conduction. 
nicely to the results obtained from $G(T)$ which are indicated as insets in Fig. 1. However, close to room temperature the slopes approach almost 0.5 while the temperature dependence of the conductance yielded $s \approx 0.8$. The reason for these differences becomes clear when the pure ac conductivity is plotted, namely $G_{\mathrm{ac}}=\left(G-G_{\mathrm{dc}}\right)$ (inset in Fig. 2). Obviously, a relaxation mode contributes to the frequency-dependent conductivity in the audiofrequency range at high temperatures $(T>200 \mathrm{~K})$. After a rough correction of the relaxation mode, as indicated by the dashed line in the inset of Fig. 2, the slope $s$ reveals the correct order of magnitude.

To get closer insight into this relaxational behavior we plotted the real [Fig. 3(a)] and the imaginary part [Fig. 3(b)] of the capacitance in Fig. 3. The imaginary part has been corrected for the dc conductivity, namely $\tilde{C}_{2}$ $=\left(G-G_{\mathrm{dc}}\right) / \omega$. The relaxational behavior, a dispersion step in the real part and a loss peak in the imaginary part, are well documented. The width of the loss peak is approximately two decades on a logarithmic frequency scale,

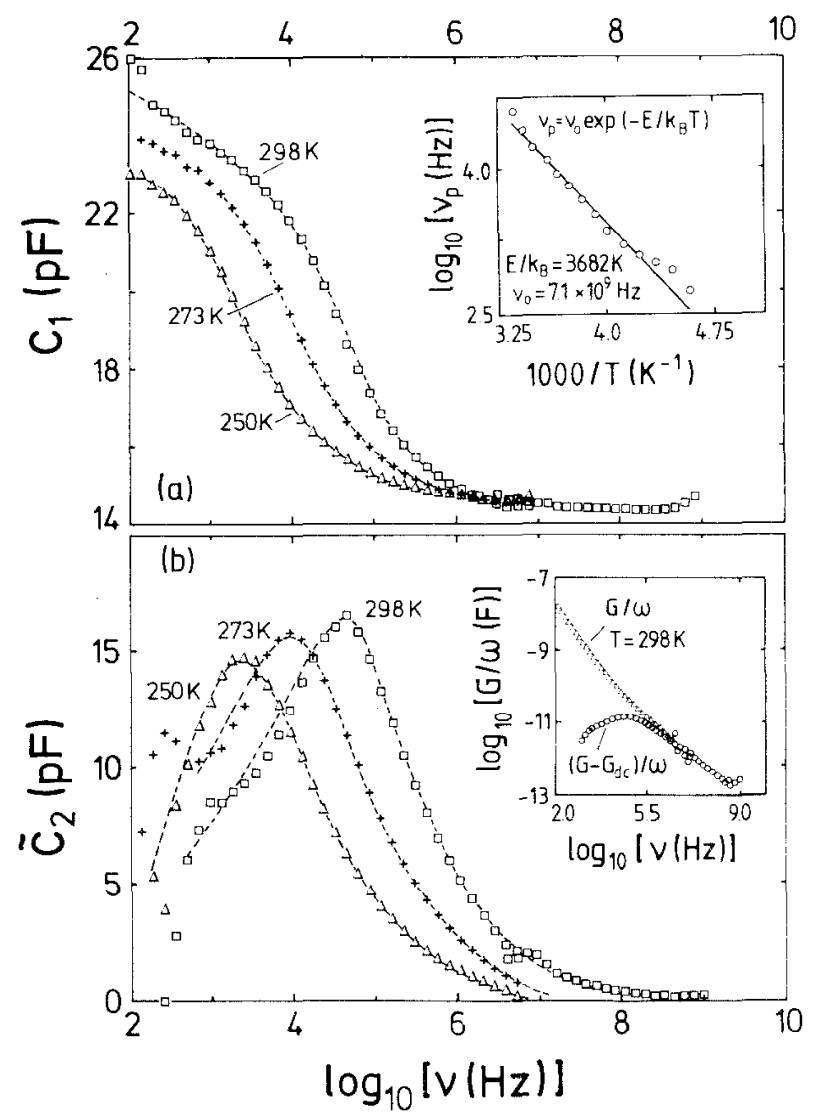

FIG. 3. Frequency dependence of the complex dielectric permittivity $C=C_{1}+i C_{2}$. (a) The real part $C_{1}$ and (b) the imaginary part of the dielectric constant $\tilde{C}_{2}=\left(G-G_{\mathrm{dc}}\right) / \omega$, corrected for the dc conductance, are plotted vs $\log _{10} v: 298 \mathrm{~K}(\square) ; 273 \mathrm{~K}$ $(+)$; and $250 \mathrm{~K}(\Delta)$. The lines are drawn to guide the eye. The inset in (b) shows raw and corrected values of the imaginary part of the dielectric constant, $G / \omega$ and $\left(G-G_{\mathrm{dc}}\right) / \omega$, respectively, vs $\log _{10} v$ at $298 \mathrm{~K}$. The inset in (a) gives the frequency of the maximum of the dielectric loss $v_{p}$ vs the inverse temperature. The solid line has been calculated using an Arrhenius-type expression $v_{p}=v_{0} \exp \left(E / k_{B} T\right)$, with $E / k_{B}=3682 \mathrm{~K}$ and $v_{0}=7.1$ $\times 10^{9} \mathrm{~Hz}$. which is slightly enhanced as compared to the Debye behavior predicting a width of 1.14 decades. This enhanced width indicates a distribution of relaxation times. The inset of Fig. 3(a) shows the frequency of the maximum loss $v_{p}$ versus the inverse temperature. The straight line was calculated assuming thermally activated behavior, $v=v_{0} \exp \left(-E / k_{B} T\right)$, with an energy barrier $E / k_{B}=3680$ $\mathrm{K}$ and an attempt frequency $v_{0}=7.1 \times 10^{9} \mathrm{~Hz}$. Relaxation phenomena in pure $\mathrm{NiO}$ single crystals have previously been reported by Kolber and MacCrone ${ }^{14}$ and were interpreted using Holstein's ${ }^{15}$ nonadiabatic limit of a hopping process of small polarons. However, within the framework of the $\mathrm{CBH}$ model a plausible and natural interpretation evolves: In the $\mathrm{CBH}$ model an assumption is made that the pair states exhibit a random spatial distribution and that pairs will contribute to the ac conductance however large their spatial extent. This assumption is unphysical and in reality there must be a cutof distance of pairs $R_{0}$ at large pair separation $R$. This cutoff $R_{0}$ defines a critical frequency $v_{c}=\omega_{c} / 2 \pi$ at which the effects of the cut-off become apparent. $6,8-10$

In the $\mathrm{CBH}$ model, in the narrow-band limit, $v_{c}$ is thermally activated and the height of the loss peak is temperature independent. ${ }^{10}$ The dielectric constant for frequencies well below the critical frequency is independent of frequency and scales almost linearly with temperature. ${ }^{10}$ All of these model predictions are in rough agreement with the experimental results of Fig. 3. (One has to keep in mind that large uncertainties in the dielectric loss are introduced due to the subtraction of the dc conductivity. Concerning the real part of the dielectric constant we neglected any temperature dependence of the infinite dielectric constant $\epsilon_{\infty}$.) Usually it is assumed that the cut-off frequency corresponds to a size $R_{0}$ beyond which the probability of finding a pair of sites becomes negligibly small. An estimate of $R_{0}$ using the energy barrier of 0.3 $\mathrm{eV}$ yields values well below $10 \AA$, which seems to be much too small.

Our results in the as-deposited $\mathrm{NiO}$ films provide clear evidence that the conduction is due to a thermal excitation of the mobility of charge carriers. The temperature and the frequency dependence of the conductivity and, in addition, the relaxation behavior at audio frequencies can consistently be explained in the CBH model. The energy difference between the ground state of the impurity level and the valence band has been determined to be $1.9 \mathrm{eV}$. Presumably, trapped holes at the nickel vacancies are the localized charge carriers. The optical energy gap of pure $\mathrm{NiO}$ is of the order of $4 \mathrm{eV}$. Thus truly intrinsic conduction is not an important factor below $1000 \mathrm{~K}$. At this point it seems interesting to note that in scandium oxide films $W_{m}$ is also of the order of $1.9 \mathrm{eV}^{6}$

We believe that these results are also important for "pure" single crystals of $\mathrm{NiO}$. In these samples the dc conduction increases exponentially but the activation temperature depends on temperature, reaching from $0.004 \mathrm{eV}$ at $10 \mathrm{~K}$ to $1 \mathrm{eV}$ at $1000 \mathrm{~K}^{4}$ At low temperatures we assume that also in the single crystals the $\mathrm{CBH}$ model gives the correct description.

Finally, we would like to comment on the observation of bound polarons at high frequencies that have been report- 
ed in the literature. ${ }^{16,17}$ The hopping of bound polarons around nickel vacancies is compatible with our data. The frequency dependence of the conductivity of bound polarons is given by ${ }^{16}$

$$
\sigma(\omega)-\sigma_{\mathrm{dc}}-\omega^{2} \tau /\left(1+\omega^{2} \tau^{2}\right)
$$

Here $\tau$ is the average time of stay between successive hops. This hopping motion around cation vacancies has been observed by Snowden and Saltsburg ${ }^{16}$ in single crystals of $\mathrm{NiO}$ and by Kabashima and Kawakubo ${ }^{17}$ in Lidoped single crystals. In both compounds a mean relaxa- tion time $\tau$ of $10^{-10} \mathrm{~s}$ has been observed. The frequency dependence of $G$ as shown in Fig. 2 reveals a strong increase of the conductivity for frequencies $v>10^{8} \mathrm{~Hz}$, with a slope of almost 2 . This strong increase might indicate a transition into the hopping regime of bound polarons as given above and in accordance with the published data. $^{17}$

This research was supported by the Materials Research Center Mainz and by the Federal Ministry for Research and Technology under Contract No. 13 N 5705/0.
${ }^{1}$ For a review, see B. H. Brandow, Adv. Phys. 26, 651 (1977); for recent developments in band-structure calculations of transition-metal monoxides, see M. R. Norman, Phys. Rev. Lett. 64, 1162 (1990); K. Terakura, A. R. Williams, T. Oguchi, and J. Kübler, ibid. 52, 1830 (1984).

${ }^{2}$ C. G. Shull, W. A. Strauser, and E. O. Wollan, Phys. Rev. 83, 333 (1951); W. L. Roth, ibid. 110, 1333 (1958).

${ }^{3}$ G. A. Slack, J. Appl. Phys. 31, 1571 (1960).

${ }^{4}$ N. F. Mott and E. A. Davis, in Electronic Processes in NonCrystalline Materials (Clarendon, Oxford, 1979); I. G. Austin and N. F. Mott, Adv. Phys. 18, 41 (1969); D. Adler, in Solid State Physics: Advances in Research and Applications, edited by F. Seitz, D. Turnbull, and H. Ehrenreich (Academic, New York, 1968), Vol. 21, p. 1; J. Appel, ibid., p. 193; A. J. Bosman and H. J. van Daal, Adv. Phys. 19, 1 (1970); D. Adler and J. Feinleib, Phys. Rev. B 2, 3112 (1970).

${ }^{5}$ K. Bange and T. Gambke, Adv. Mater. 2, 10 (1990), and references therein.

${ }^{6}$ G. E. Pike, Phys. Rev. B 6, 1572 (1972).

${ }^{7}$ M. Pollak and G. E. Pike, Phys. Rev. Lett. 28, 1449 (1972).

${ }^{8}$ S. R. Elliott, Philos. Mag. 36, 1291 (1977); 37, 553 (1978).
${ }^{9}$ S. R. Elliott, Adv. Phys. 36, 135 (1987).

${ }^{10}$ A. R. Long, Adv. Phys. 31, 553 (1982).

${ }^{1} \mathrm{~K}$. Bange, U. Martens, A. Nemetz, and A. Temmink, in Electrochromic Materials, Proceedings of the Physical Electrochemistry Division, edited by M. K. Carpenter and D. A. Corrigan (Electrochemical Society, Pennington, NJ, 1990), Vol. 90-2, p. 334; C. R. Ottermann, A. Temmink, and K. Bange, Thin Solid Films 193/194, 409 (1990); A. Nemetz, Diplomarbeit, Universität Mainz, Mainz, 1990 (unpublished).

${ }^{12}$ R. Böhmer, M. Maglione, P. Lunkenheimer, and A. Loidl, J. Appl. Phys. 65, 901 (1989).

13. . E. Keem, I. M. Honig, and I. I. van Zandt, Philos. Mag. B 37, 537 (1978).

${ }^{14}$ M. A. Kolber and R. K. MacCrone, Phys. Rev. Lett. 29, 1457 (1972).

${ }^{15}$ T. Holstein, Ann. Phys. (N.Y.) 8, 343 (1959).

${ }^{16}$ D. P. Snowden and H. Saltsburg, Phys. Rev. Lett. 14, 497 (1965).

${ }^{17}$ S. Kabashima and T. Kawakubo, J. Phys. Soc. Jpn. 24, 493 (1968). 